\title{
Characteristics of Cognitive Status in Sub-Population of Sub- Acute Stage of Ischemic Stroke Patients in West Nusa Tenggara, Indonesia
}

\author{
Herpan Syafii Harahap 1,2*, Muhammad Akbar³, Jumraini Tammasse³, Andi Kurnia Bintang ${ }^{3}$, Andi Alfian Zainuddin ${ }^{4}$
}

${ }^{1}$ Doctoral Program, Faculty of Medicine Universitas Hasanuddin, Makassar, Indonesia, 2 Department of Neurology, Faculty of Medicine, Universitas Mataram, Mataram, Indonesia, ${ }^{3}$ Department of Neurology, Faculty of Medicine, Universitas Hasanuddin, Makassar, Indonesia, ${ }^{4}$ Department of Public Health, Faculty of Medicine, Universitas Hasanuddin, Makassar, Indonesia

\begin{abstract}
Cognitive decline is a significant complication that affects most stroke survivors. Early detection of cognitive decline in ischemic stroke patients and identification of risk factors improves their clinical outcomes. This study aimed to determine the characteristics of cognitive status in the sub-acute phase of ischemic stroke. A cross-sectional study was conducted on 89 sub-acute ischemic stroke patients in three hospitals in West Nusa Tenggara recruited consecutively from August 2019 to April 2020. The data collected were demographic and clinical characteristics, cognitive status, and functional outcome. The association between clinical and demographic characteristics and cognitive decline was analyzed using logistic regression. In addition, the relationship between cognitive status and functional outcomes of these patients was examined using the chi-square test. This study revealed that the prevalence of cognitive decline in these subjects was $71.9 \%$. Multiple logistic regression showed that age was the only characteristic associated with cognitive decline in the subjects $(\mathrm{OR}=5.12$, $95 \% \mathrm{Cl}=1.08-24.28)$. Furthermore, the frequency of cognitive decline in these subjects was significantly associated with functional outcomes $(p-v a l u e=$ 0.014). Thus, there was a high prevalence of cognitive decline in sub-acute ischemic stroke patients associated with increasing age and poor functional outcomes.
\end{abstract}

Keywords: brain ischemia, cognitive dysfunction, neuropsychological tests, stroke

\section{Introduction}

Cognitive decline is considered an essential complication of ischemic stroke, increasing to $80 \%$ in a few weeks post-stroke. ${ }^{1}$ It may involve one or more cognitive domains ranging from mild to that which interferes with functional capacities, regardless of their neurological deficits. $^{2}$ Approximately 59\% of mild ischemic stroke patients of productive age and higher education level remain unable to return to work after six months of followup due to stroke-related cognitive decline. ${ }^{3}$ On long-term evaluation, about $30 \%$ of ischemic stroke patients with cognitive decline tend to be stable in the first four years or even progress to dementia if not appropriately treated. ${ }^{4}$ These patients will eventually increase the health, social, and economic burden on their careers, families, and the healthcare system. ${ }^{5}$ Meanwhile, early intervention of cognitive decline after ischemic stroke may delay its progression to dementia and provide better clinical outcomes. ${ }^{6}$ Therefore, early evaluation of cognitive function in ischemic stroke patients, especially in the subacute phase, is important.

Correspondence $^{*}$ : Herpan Syafii Harahap, Department of Neurology Faculty of Medicine Universitas Mataram, Pendidikan Street No.37 Mataram 83125, Indonesia, E-mail: herpanharahap@unram.ac.id, Phone: +62-817 5770062
Typically, older age, lower educational level, hypertension, diabetes mellitus, atrial fibrillation, brain region affected, and size and location of infarction are the clinical and demographic characteristics known as risk factors for cognitive decline after ischemic stroke. ${ }^{7}$ Older age is the most important independent risk factor for the cognitive decline from all causes, including stroke. ${ }^{6}$ Among the well-identified vascular risk factors, hypertension and diabetes mellitus are the most critical risk factors for cognitive decline in the general population. ${ }^{8}$ Therefore, in most cases, it is difficult to ascertain whether the cognitive decline observed in patients after ischemic stroke is solely due to an ischemic stroke event. However, cognitive decline after ischemic stroke is predominantly due to interactions between the ischemic stroke event and risk factors for cognitive decline after ischemic stroke. ${ }^{1}$ Therefore, the intervention of the risk factors for ischemic stroke must also be considered to manage cognitive decline after ischemic stroke.

Regarding the evaluation of cognitive function among ischemic stroke patients, previous studies had employed

Received : October 6, 2020

Accepted : April 29, 2021

Published: August 242021 
diverse research methods, determining the appropriate time frame and selection of instruments for the assessment of cognitive function. ${ }^{2}$ Previous studies also showed diversity in the ethnicity of the subjects, which could increase the variability of the demographic characteristics of the patients and modified their susceptibility to cognitive decline after ischemic stroke. ${ }^{9}$ However, similar studies that have evaluated the cognitive functions among these patients remained scarce, including those in Indonesia.

Determining the appropriate time for evaluating cognitive function among ischemic stroke patients to provide relevant results to be used as a basis for early intervention in cognitive decline in these subjects is also important. Several studies have evaluated the acute and sub-acute outcomes beyond three months after the onset of ischemic stroke, with or without the follow-up of the progression of cognitive decline. ${ }^{10,11}$ The primary data collection on cognitive status and cognitive decline early detection in post-ischemic stroke patients is mandatory. It is based on cognitive decline after the ischemic stroke that can improve due to activities of neural plasticity in the brain that generally occur optimally in the first three months. ${ }^{12}$ In the acute stage of ischemic stroke, patients can experience delirium due to ischemic stroke-associated cerebral edema and the involvement of the right hemisphere, affecting patients' performance in the evaluation of cognitive function. ${ }^{13,14}$ Since cerebral edema generally undergoes resolution within the first weeks, the assessment of cognitive disorders should be performed in the sub-acute stage. 15

This study aimed to investigate the cognitive status of the sub-acute stage in patients with ischemic stroke. This is the first study conducted in a sub-population of ischemic stroke patients in the sub-acute stage of rural areas in West Nusa Tenggara to represent the rural regions in Indonesia. The results of this study are essential for local health authorities to assess the importance of early detection and management of cognitive decline in the sub-acute stage of ischemic stroke.

\section{Method}

This study employed a cross-sectional design that involved ischemic stroke patients in the sub-acute stage consecutively recruited in Nusa Tenggara Barat General Hospital, Mataram General Hospital, and Siti Hajar Hospital. This study was conducted between August 2019 and April 2020. The sample size was calculated using the sample calculation formula $(\mathrm{Z} \alpha 2 \mathrm{PQ}) / \mathrm{d}^{2}$. Since $\alpha$ $=0.05, \mathrm{Z} \alpha=1.96, \mathrm{P}$ (prevalence) $=70 \%, \mathrm{Q}=1-\mathrm{P}$, and $\mathrm{d}$ (margin of error) $=10 \%$, the sample size was 81 . Eight samples ( $10 \%$ of the calculated sample size) were added; thus, the final sample size of the study was 89 .

The inclusion criteria for the subjects were ischemic stroke confirmed by head computed tomography (CT) in the sub-acute stage (2-12 weeks after stroke onset). The subjects were fully conscious, aged $40-70$ years, with at least a primary school graduation (6-year-education). The age of the subjects was determined to be between 40-70 years to minimize the confounding effect of the possibility of cognitive decline related to neurodegenerative processes in patients above 70 years of age. ${ }^{16}$ This study employed a cognitive evaluation instrument that required patient awareness of the concept of time and aspects of knowledge. This concept was only obtained when the patient reached the fourth grade and was established at the sixth grade of elementary school education level. For this reason, the subjects were selected to have a minimum education level of primary school graduates.

Meanwhile, the exclusion criteria for the subjects were patients with significant vision and hearing disorders that could not be corrected, prior history of dementia, psychiatric disorders, benzodiazepines, antipsychotics, and antidepressant medication at the time of cognitive evaluation. This study was approved by the Health Research Ethics Commission of Universitas Mataram (Register number: 214/UN18.F7/ETIK/2019). All subjects provided written informed consent before participation.

Demographic and clinical variables collected in this study were age, gender, level of education, side of the lesion in the brain based on head CT scan, infarction size, hypertension, diabetes mellitus, cigarette smoking, atrial fibrillation, cognitive status, and functional outcomes. Before data analysis, categorization of both demographic and clinical characteristics was performed. In terms of the demographic characteristics of the subjects, age was categorized as young adults (40-59 years) and older (6070 years); gender was categorized as male or female, and level of education was categorized as elementary school, high school, and college.

Based on the head CT scan examination results, the side of the lesion in the brain was classified as the right hemisphere, left hemisphere, and bilateral. In contrast, infarction size was categorized into small $(\leq 5 \mathrm{~mm}$ in diameter), medium (6-14 $\mathrm{mm}$ in diameter), and large ( $\geq 15$ $\mathrm{mm}$ in diameter), which was a modification of the categories used in previous studies. ${ }^{17,18}$ Subjects were categorized as having hypertension if they had a systolic blood pressure of $\geq 140 \mathrm{mmHg}$, and/or diastolic blood pressure $\geq 90 \mathrm{mmHg}$, and/or any use of antihypertensive medication, and/or self-reported history of hypertension. They were categorized as having diabetes mellitus if they had fasting blood glucose levels of $\geq 126 \mathrm{mg} / \mathrm{dL} \quad(7.0$ $\mathrm{mmol} / \mathrm{L}$ ), and/or any use of anti-diabetic medication, and/or self-reported history of diabetes mellitus as described in previous studies. ${ }^{19,20}$ In terms of cigarette smoking, subjects were categorized as smokers and non- 
smokers. Twelve-lead electrocardiogram (ECG) examinations were performed on all subjects, and an independent cardiologist reviewed the results to identify the presence of atrial fibrillation.

Cognitive status was determined based on the grade of the Montreal Cognitive Assessment in the Indonesian version (MoCA-Ina) score. The MoCA-Ina is an instrument for evaluating global cognitive functions that have been validated for Indonesian populations. ${ }^{21}$ Its total score ranges from $0-30$. Subjects with a score of 26-30 were categorized as normal, while those with 0-25 were indicated to have a cognitive decline. Subjects with an education time of $\leq 12$ years were given an additional score of 1 as a correction factor for the effects of education.

The functional outcome of patients was established based on the Barthel index (BI) score. The Barthel index is a valuable instrument for assessing the functional outcome of subjects with ischemic stroke regarding their independence in carrying out basic daily activities. ${ }^{22}$ This instrument has a score in the range of 0-100. Subjects with a score of $80-100$ were categorized as functionally independent, while those with 0-79 were classified as functionally dependent as described in the previous study. 23

The first analysis was carried out to verify the association between demographic and clinical variables and cognitive status in the sub-acute stage of ischemic stroke.
In this step, the association between categorical independent variables, including age, gender, level of education, side of the lesion in the brain, infarction size, hypertension, diabetes mellitus, cigarette smoking, atrial fibrillation, and cognitive status of the subjects were initially analyzed using simple binary logistic regression and crude odds ratio (OR) with $95 \%$ confidence interval (CI). In the second analysis, the independent variables (p-value $<0.25$ in simple binary logistic regression analysis) were then grouped into multiple logistic regression models and had the OR adjusted with 95\% CI. The third analysis was administered using the chi-square test to ascertain the relationship between these subjects' cognitive status and functional outcomes. Statistical significance was set at p-value $<0.05$.

\section{Results}

Table 1 shows the results of simple binary logistic regression analysis examining the association between demographic and clinical variables and cognitive decline among subjects with ischemic stroke in the sub-acute stage $(n=89)$. This analysis revealed that age group and infarction size were the eligible variables for the final model of multiple logistic regression ( $\mathrm{p}$-value $<0.25$ ). Although only age had a significant association (OR = $4.86,95 \% \mathrm{CI}=1.04-22.68)$, medium $(\mathrm{OR}=3.17,95 \%$ $\mathrm{CI}=0.69-14.46)$ and large infarct size $(\mathrm{OR}=3.06,95 \%$ $\mathrm{CI}=0.78-11.10)$ also appeared to modulate an increased

Table 1. Simple Binary Logistic Regression Analysis Showing Variables Associated with Cognitive Decline in the Sub-Acute Stage of Ischemic Stroke

\begin{tabular}{|c|c|c|c|c|c|c|c|}
\hline \multirow{3}{*}{ Variable } & \multirow{3}{*}{ Category } & \multicolumn{4}{|c|}{ Cognitive Status } & \multirow{3}{*}{ Crude OR $(95 \%$ CI $)$} & \multirow{3}{*}{ p-value } \\
\hline & & \multicolumn{2}{|c|}{ Normal (n = 25) } & \multicolumn{2}{|c|}{ Decline $(n=64)$} & & \\
\hline & & $\mathbf{n}$ & $\%$ & $\mathbf{n}$ & $\%$ & & \\
\hline \multirow[t]{2}{*}{ Age group } & Older & 2 & 9.5 & 19 & 90.5 & $4.86(1.0-22.68)$ & $0.040^{*}$ \\
\hline & Young adult & 23 & 33.8 & 45 & 66.2 & Reference & \\
\hline Gender & Female & 5 & 20.0 & 20 & 80.0 & Reference & \\
\hline \multirow{3}{*}{ Level of education } & College & 8 & 27.6 & 21 & 72.4 & $1.31(0.4-3.76)$ & 0.612 \\
\hline & High school & 13 & 33.3 & 26 & 66.7 & $0.62(0.1-2.41)$ & 0.487 \\
\hline & Elementary school & 4 & 19.0 & 17 & 81.0 & Reference & \\
\hline \multirow[t]{3}{*}{ Side of the lesion } & Right hemisphere & 10 & 27.8 & 26 & 72.2 & $1.04(0.29-3.69)$ & 0.952 \\
\hline & Left hemisphere & 10 & 28.6 & 25 & 71.4 & $1.00(0.28-3.54)$ & 1.000 \\
\hline & Bilateral & 5 & 27.8 & 13 & 72.2 & Reference & \\
\hline \multirow[t]{2}{*}{ Infarction size } & Large $(\geq 15 \mathrm{~mm})$ & 3 & 13.6 & 19 & 86.4 & $3.17(0.69-14.46)$ & $0.137 *$ \\
\hline & Small $(\leq 5 \mathrm{~mm})$ & 15 & 32.6 & 31 & 67.4 & Reference & \\
\hline \multirow[t]{2}{*}{ Hypertension } & Yes & 21 & 26.2 & 59 & 73.8 & $0.44(0.11-1.82)$ & 0.259 \\
\hline & No & 4 & 44.4 & 5 & 55.6 & Reference & \\
\hline \multirow[t]{2}{*}{ Diabetes mellitus } & Yes & 9 & 26.5 & 25 & 73.6 & $0.88(0.34-2.29)$ & 0.789 \\
\hline & No & 16 & 29.1 & 39 & 70.9 & Reference & \\
\hline \multirow[t]{2}{*}{ Cigarette smoking } & Smokers & 12 & 34.3 & 23 & 65.7 & $1.64(0.64-4.20)$ & 0.297 \\
\hline & Non-smokers & 13 & 24.1 & 41 & 75.9 & Reference & \\
\hline \multirow[t]{2}{*}{ Atrial fibrillation } & Yes & 0 & 0.0 & 7 & 100.0 & $0.00(0.00-0.00)$ & 0.999 \\
\hline & No & 25 & 30.5 & 57 & 69.5 & Reference & \\
\hline
\end{tabular}

Notes: *eligible for multiple logistic regression analysis, OR: Odds Ratio, CI: Confidence Interval 
Table 2. The Final Model of Multiple Logistic Regression Showing Eligible Variables Associated with Cognitive Decline in the Sub-Acute Stage of Ischemic Stroke

\begin{tabular}{|c|c|c|c|c|c|c|c|}
\hline \multirow{3}{*}{ Variable } & \multirow{3}{*}{ Category } & \multicolumn{4}{|c|}{ Cognitive Status } & \multirow{3}{*}{ Crude OR (95\% CI) } & \multirow{3}{*}{ p-value } \\
\hline & & \multicolumn{2}{|c|}{ Normal $(n=25)$} & \multicolumn{2}{|c|}{ Decline $(n=64)$} & & \\
\hline & & $\mathbf{n}$ & $\%$ & $\mathbf{n}$ & $\%$ & & \\
\hline \multirow[t]{2}{*}{ Age group } & Older & 2 & 9.5 & 19 & 90.5 & $5.12(1.08-24.28)$ & $0.040^{*}$ \\
\hline & Young adult & 23 & 33.8 & 45 & 66.2 & Reference & \\
\hline \multirow[t]{3}{*}{ Infarction size } & Large $(\geq 15 \mathrm{~mm})$ & 3 & 13.6 & 19 & 86.4 & $3.38(0.72-15.95)$ & 0.124 \\
\hline & Medium $(6-14 \mathrm{~mm})$ & 7 & 33.3 & 14 & 66.7 & $3.27(0.81-13.12)$ & 0.095 \\
\hline & Small $(\leq 5 \mathrm{~mm})$ & 15 & 32.6 & 31 & 67.4 & Reference & \\
\hline
\end{tabular}

Notes: *significant association, OR: Odds Ratio, CI: Confidence Interval

Table 3. The Association between Cognitive Status and Functional Outcomes of the Subjects with Sub-Acute Stage of Ischemic Stroke

\begin{tabular}{|c|c|c|c|c|c|}
\hline \multirow{3}{*}{ Functional Outcome } & \multicolumn{4}{|c|}{ Cognitive Status } & \multirow{3}{*}{ p-value } \\
\hline & \multicolumn{2}{|c|}{ Normal $(n=25)$} & \multicolumn{2}{|c|}{ Decline $(n=64)$} & \\
\hline & $\mathbf{n}$ & $\%$ & $\mathrm{n}$ & $\%$ & \\
\hline Functionally independent & 25 & 32.5 & 52 & 67.5 & $0.014 *$ \\
\hline Functionally dependent & 0 & 0.0 & 12 & 100.0 & \\
\hline
\end{tabular}

Notes: *significant difference ( $\mathrm{p}$-value $<0.05$ ), chi-squared test

risk of ischemic stroke-associated cognitive decline.

Table 2 shows the results of the final model of multiple logistic regression analysis in examining the association between eligible variables and cognitive decline among subjects with ischemic stroke in the sub-acute stage $(n=89)$. This final model of multiple logistic regression revealed that age was the only variable associated with cognitive decline in the subjects $(\mathrm{OR}=5.12$, $95 \% \mathrm{CI}=1.08-24.28)$. Consistent with the simple logistic regression analysis results presented previously, older subjects had a greater risk of ischemic stroke-associated cognitive decline.

Table 3 shows the chi-square test results in investigating the association between cognitive status and functional outcomes of ischemic stroke patients in the subacute stage. The chi-square test revealed that cognitive status was associated with the clinical outcomes of the subjects $(p$-value $=0.014)$. Subjects with ischemic stroke-associated cognitive decline had higher functional dependence than those with normal cognitive status.

\section{Discussion}

This initial study aimed to investigate the characteristics of cognitive status in the sub-acute stage of ischemic stroke in West Nusa Tenggara, representing rural areas in Indonesia. This study showed a high prevalence of cognitive decline in the sub-acute stage of ischemic stroke, amounting to $71.9 \%$. This result was in line with the findings of previous studies. ${ }^{24,25}$ Therefore, this result added to previous data on the prevalence of ischemic stroke-associated cognitive impairment. Since the majority of ischemic stroke continues to increase with higher survival rates in its patients, including in Indonesia, the results of this study suggested that ischemic stroke-associated cognitive decline has the potential to become a significant health problem in the future. The relatively consistent data on the prevalence of ischemic stroke-associated cognitive decline between this study and previous studies also suggested that ischemic stroke-associated cognitive decline will also become a global health problem, both in urban and rural areas. Suppose an adequate management strategy is not developed earlier. In that case, this health problem has the potential to cause a decrease in their productivity and an increase in the economic burden of their families and the existing healthcare system.

This study also showed that increasing age was the only variable significantly associated with cognitive decline in the sub-acute stage of ischemic stroke in West Nusa Tenggara. The results of this study were in accordance with previous studies, which showed that increasing age was the most substantial risk factor for poststroke cognitive decline, including in ischemic stroke. 6,26 Since patients over 70 years old were excluded from this study, the role of neurodegenerative processes predominantly involving brain amyloid-beta $(\mathrm{A} \beta)$ deposition in 
the presence of a cognitive decline in patients with subacute ischemic stroke is unlikely. The negative impact of increasing age on cognitive flexibility needs to be considered to play an important role in the pathophysiology of cognitive decline after stroke. Povroznik, et al., ${ }^{27}$ showed that increasing age exacerbates stroke-related cognitive flexibility deficits, leading to cognitive decline in stroke patients. Since the success of cognitive impairment interventions is primarily determined by the patients' ability to understand the instructions given during the intervention program, the management of ischemic stroke-associated cognitive decline associated with ischemic stroke in elderly patients will be a challenge for existing healthcare providers in the province of West Nusa Tenggara. Therefore, these results can be considered as a basis for the development of intervention strategies for ischemic stroke-associated cognitive decline by health authorities in this province.

However, other variables, including gender, level of education, lesion side in the brain, infarction size, hypertension, diabetes mellitus, cigarette smoking, and atrial fibrillation, were not related to cognitive decline in the patients. In general, the level of education of the population living in rural areas is lower than that of those living in urban areas, which will cause a higher prevalence of cognitive decline after ischemic stroke in rural areas compared to those in urban areas. ${ }^{10}$ Nonetheless, previous studies investigating the risk factors for cognitive decline in ischemic stroke, including increasing age, level of education, ethnicity, geography, hypertension, diabetes mellitus, brain region affected, cardioembolic type of ischemic stroke, and the size and location of infarction had shown varied results. Zulkifly, et al., ${ }^{7}$ showed that the level of education, hypertension, diabetes mellitus, brain region affected, and the size and location of infarction were variables related to cognitive decline after stroke. In contrast, Levine, et al., 28 showed that age and cardioembolic stroke were the main risk factors for cognitive decline. These varying results were determined primarily by the study population, methods, and cognitive evaluation tools used. Nevertheless, intervention on modifiable vascular risk factors remains an essential part of the management strategy for cognitive decline after ischemic stroke.

This study also indicated that the cognitive status of the sub-acute stage of ischemic stroke was associated with their functional outcome measured using the Barthel Index (BI) score. The frequency of the sub-acute stage of ischemic stroke patients who were functionally dependent in performing basic activities of daily living was higher than in those with normal cognitive function. This result was also in accordance with previous studies conducted by Abzhandadze, et al., ${ }^{29}$ and Li, et al. ${ }^{22}$ Early rehabilitation therapy is the most important factor that de- termines the significance of cognitive status and functional outcomes in ischemic stroke patients. Since brain plasticity is optimally improved within the first three months, early rehabilitation therapy should be initiated during this period. Therefore, this result can also be used to develop rehabilitation strategies for stroke-related cognitive decline by local health authorities.

This study had some limitations. The first was dealing with the lack of facilities used to establish a diagnosis of cognitive decline in the sub-acute stage of ischemic stroke. Since the CT scan was the only diagnostic facility available in this study, the clinical characterization of ischemic stroke was difficult to achieve. This hinders the presentation of data on the analysis of the relationship between clinical characteristics and cognitive decline in ischemic stroke patients in more detail. The second was the absence of baseline data on cognitive status prior to stroke events from the subjects in this study. It was difficult to determine whether this cognitive decline was from a pre-existing condition or as a result of an ischemic stroke event.

Nevertheless, the results of this study can be used as a basis for developing detection and intervention strategies by local health authorities in West Nusa Tenggara and other parts of Indonesia having similar characteristics. It was based on data for the prevalence of cognitive decline associated with sub-acute ischemic stroke, and its risk factors were still very limited, especially among Indonesian populations living in rural regions. A longitudinal study aimed at investigating the benefits of early intervention in the sub-acute stage of ischemic stroke patients on cognitive improvement and functional outcomes is needed.

\section{Conclusion}

The present study revealed a high prevalence of cognitive decline in the sub-acute stage of ischemic stroke. Age was the only variable significantly associated with cognitive decline. The sub-acute stage of ischemic stroke in patients with increasing age has a higher risk of cognitive decline. The high prevalence of cognitive decline in these subjects was associated with poor functional outcomes. These results add to previous data regarding the characteristics of stroke-associated cognitive decline in Indonesia and other developing countries. More importantly, these results can be used as a basis for developing strategies for early detection, intervention, and rehabilitation of stroke-related cognitive decline in West Nusa Tenggara and other regions in Indonesia with similar characteristics.

\footnotetext{
Abbreviations

CT: Computed Tomography; ECG: Electrocardiogram; MoCA-Ina: Montreal Cognitive Assessment in Indonesian version; BI: Barthel
} 
Index; $\mathrm{A} \beta$ : Amyloid-Beta.

\section{Ethics Approval and Consent to Participate}

This study was approved by the Health Research Ethics Commission of Universitas Mataram, Mataram (Register number: 214/UN18.F7/ETIK/2019). All subjects provided were informed under written consent prior to their participation.

\section{Competing Interest}

The author declares that there is no significant competing financial, professional, or personal interest that might have affected the performance or presentation of the work described in this manuscript.

\section{Availability of Data and Materials}

Data and Materials of the present study are available from the corresponding author for reasonable request and non-commercial purposes.

\section{Authors' Contribution}

HSH, MA, JT, AKB, and AAZ conceptualized and designed the study, analyzed and interpreted the study results. HSH drafted the manuscript. $\mathrm{HSH}, \mathrm{MA}, \mathrm{JT}, \mathrm{AKB}$, and AAZ revised the manuscript.

\section{Acknowledgment}

The authors would like to acknowledge the hospital staff of Nusa Tenggara Barat General Hospital, Mataram General Hospital, and Siti Hajar Hospital that supported this study.

\section{References}

1. Sun J-H, Tan L, Yu J-T. Post-stroke cognitive impairment: epidemiology, mechanisms and management. Annals of Translational Medicine. 2014; 2 (8): 80.

2. Melkas S, Jokinen H, Hietanen M, Erkinjuntti T. Poststroke cognitive impairment and dementia: prevalence, diagnosis, and treatment. Degenerative Neurological and Neuromuscular Disease. 2014; 4: 21 7.

3. Kauranen T, Turunen K, Laari S, Mustanoja S, Baumann P, Poutiainen E. The severity of cognitive deficits predicts return to work after a first-ever ischaemic stroke. Journal of Neurology, Neurosurgery, and Psychiatry. 2013; 84: 316-21.

4. Makin SDJ, Turpin S, Dennis MS, Wardlaw JM. Cognitive impairment after lacunar stroke: systematic review and meta-analysis of incidence, prevalence and comparison with other stroke subtypes. Journal of Neurology, Neurosurgery, and Psychiatry. 2013; 84: 893-900.

5. Rohde D, Williams D, Gaynor E, Bennett K, Dolan E, Callaly E, et al. Secondary prevention and cognitive function after stroke: a study protocol for a 5-year follow-up of the ASPIRE-S cohort. BMJ Open. 2017; 7: e014819.

6. Kalaria RN, Akinyemi R, Ihara M. Stroke injury, cognitive impairment and vascular dementia. Biochimica et Biophysica Acta - Molecular Basis of Disease. 2016; 1862 (5): 915-25.

7. Zulkifly MFM, Ghazali SE, Din NC, Singh DKA, Subramaniam P. A review of risk factors for cognitive impairment in stroke survivors. The Scientific World Journal. 2016; 2016: 3456943.

8. Takeda S, Rakugi H, Morishita R. Roles of vascular risk factors in the pathogenesis of dementia. Hypertension Research. 2020; 43: 162-7.

9. Lo JW, Crawford JD, Desmond DW, Godefroy O, Jokinen H, Mahinrad S, et al. Profile of and risk factors for poststroke cognitive impairment in diverse ethnoregional groups. Neurology. 2019; 93: e2257-71

10. Danovska M, Stamenov B, Alexandrova M, Peychinska D. Post-stroke cognitive impairment - phenomenology and prognostic. Journal of International Medical Association Bulgaria. 2012; 18: 290-7.

11. Obaid M, Flach C, Marshall I, Wolfe CDA, Douiri A. Long-term outcomes in stroke patients with cognitive impairment: a populationbased study. Geriatrics. 2020; 5 (2): 32.

12. Lo Coco D, Lopez G, Corrao S. Cognitive impairment and stroke in elderly patients. Vascular Health and Risk Management. 2016; 12: $105-16$.

13. Balami JS, Chen R-L, Grunwald IQ, Buchan A. Neurological complications of acute ischemic stroke. The Lancet Neurology. 2011; 10 (4): 357-71.

14. Dostovic Z, Dostovic E, Smajlovic D, Ibrahimagic OC, Avdic L, Becirovic E. Predictors for post-stroke delirium outcome. Materiasocio-medica. 2016; 28 (5): 382-6.

15. Battey TWK, Karki M, Singhal AB, Wu O, Sadaghiani S, Campbell BC $\mathrm{V}$, et al. Brain edema predicts outcome after nonlacunar ischemic stroke. Stroke. 2014; 45: 3643-8.

16. Morley JE, Morris JC, Berg-Weger M, Borson S, Carpenter BD, del Campo N, et al. Brain health: the importance of recognizing cognitive impairment: an IAGG consensus conference. Journal of the American Medical Directors Association. 2015; 16 (9): 731-9.

17. Asdaghi N, Pearce LA, Nakajima M, Field TS, Bazan C, Cermeno F, et al. Clinical correlates of infarct shape and volume in lacunar strokes the SPS3 trial. Stroke. 2014; 45 (10): 2952-8.

18. He Y, Wei L, Li W. Comparison of application values of CT and MRI in the diagnosis of early lacunar infarction. Pakistan Journal of Medical Sciences. 2018; 34 (1): 190-3.

19. Lou H, Dong Z, Zhang P, Shao X, Li T, Zhao C, et al. Interaction of diabetes and smoking on stroke: a population-based cross-sectional survey in China. BMJ Open. 2018; 8: e017706.

20. Lu S, Bao M-Y, Miao S-M, Zhang X, Jia Q-Q, Jing S-Q, et al. prevalence of hypertension, diabetes, and dyslipidemia, and their additive effects on myocardial infarction and stroke: a cross-sectional study in Nanjing, China. Annals of Translational Medicine. 2019; 7 (18): 436.

21. Rambe AS, Fitri FI. Correlation between the Montreal cognitive assessment- Indonesian version (MoCA-Ina) and the Mini-mental state examination (MMSE) in elderly. Open Access Macedonian Journal of Medical Sciences. 2017; 5 (7): 915-9.

22. Li Q-X, Zhao X-J, Wang Y, Wang D-L, Zhang J, Liu T-J, et al. Value of the Barthel scale in prognostic prediction for patients with cerebral infarction. BMC Cardiovascular Disorders. 2020; 20 (1): 14.

23. Ghosal MK, Burman P, Singh V, Das S, Paul N, Ray BK, et al. Correlates of functional outcome among stroke survivors in a developing country - a prospective community-based study from India. Journal of Stroke and Cerebrovascular Diseases. 2014; 23 (10): 2614 21.

24. Yu K-H, Cho S-J, Oh MS, Jung S, Lee J-H, Shin J-H, et al. Cognitive impairment evaluated with vascular cognitive impairment harmoniza- 
tion standards in a multicenter prospective stroke cohort in Korea. Stroke. 2013; 44: 786-8.

25. Qu Y, Zhuo L, Li N, Hu Y, Chen W, Zhou Y, et al. prevalence of post-stroke cognitive impairment in China: a community-based, crosssectional study. PLoS One. 2015; 10 (4): e0122864.

26. Tang EYH, Amiesimaka O, Harrison SL, Green E, Price C, Robinson $\mathrm{L}$, et al. Longitudinal effect of stroke on cognition: a systematic review. Journal of the American Heart Association. 2018; 7 (2): e006443.

27. Povroznik JM, Ozga JE, Haar CV, Engler-Chiurazzi EB. Executive (dys) function after stroke: special considerations for behavioral pharmacology. Behavioural Pharmacology. 2018; 29 (7): 638-53.

28. Levine DA, Wadley VG, Langa KM, Unverzagt FW, Kabeto MU, Giordani B, et al. Risk factors for post-stroke cognitive decline: the REGARDS study. Stroke. 2018; 49 (4): 987-94.

29. Abzhandadze T, Rafsten L, Lundgren-Nilsson Å, Sunnerhagen KS. Feasibility of cognitive functions screened with the montreal cognitive assessment in determining ADL dependence early after stroke. Frontiers in Neurology. 2018; 9: 705. 